\title{
RUPTURA GÁSTRICA POR BAROTRAUMA
}

\author{
Barogenic rupture of the stomach
}

Rodrigo Severo de Camargo PEREIRA, Roberta Thiery Godoy ARASHIRO, Rogerio SAAD-HOSSNE

ABCDDV/614

Pereira RSC, Arashiro RTG, Saad-Hossne R. Ruptura gástrica por barotrauma. ABCD Arq Bras Cir Dig 2008;21(3):144-6

RESUMO - Introdução - A ruptura gástrica por barotrauma é uma causa rara de abdome agudo perfurativo, sendo geralmente tratada por laparotomia e rafia primária da lesão. Nas reanimações cardiopulmonares pode ocorrer 9 a $12 \%$ de lesões de mucosa gástrica. Relato do caso - Mulher no $5^{\circ}$ dia de puerpério necessitou intubação orotraqueal devido à pneumonia hospitalar. Após procedimento evoluiu com distensão abdominal importante, associada a sinais de choque séptico. Após radiografia simples de abdome foi constado pneumoperitôneo. Submetida à laparotomia exploradora evidenciou-se ruptura de pequena curvatura gástrica de $7 \mathrm{~cm}$. O tratamento da lesão foi com sutura primária. Recebeu alta no $14^{\circ}$ do pós operatório após término do tratamento para pneumonia. Conclusão - Apesar de rara, a ruptura gástrica por barotrauma deve ser sempre aventada quando após reanimação cardiopulmonar houver distensão abdominal refratária à sondagem naso-gástrica.

DESCRITORES - Barotrauma. Ruptura gástrica.

\section{INTRODUÇÃO}

A ruptura gástrica por barotrauma constitui causa rara de abdome agudo perfurativo, tendo sido encontrado poucos relatos na literatura ${ }^{1,2,3,5,6,7}$. É geralmente tratada por laparotomia exploradora e sutura primária da lesão, porém, segundo Yeung et $\mathrm{al}^{7}$, em alguns casos é possível adotar-se conduta conservadora. Pode ocorrer de 9 a $12 \%$ de lesão de mucosa gástrica após reanimação cardio-pulmonar ${ }^{5}$.

\section{RELATO DO CASO}

Mulher de 20 anos com diagnóstico de tuberculose pulmonar em uso de Isoniazida e Rifampicina e no $5^{\circ}$ dia de puerpério de parto vaginal, iniciou quadro de dispnéia, tosse produtiva e febre, tendo sido feito diagnóstico de pneumonia. Havendo piora progressiva do quadro, mesmo em vigência de antibioticoterapia de amplo espectro (Imipenem e Clindamicina), ocorreu insuficiência respiratória, sendo indicada intubação orotraqueal. Após procedimento, evoluiu com distensão abdominal importante, dificultando a ventilação, e desaparecimento da macicez hepática (sinal de Jobert), sendo constatada canulação iatrogênica do esôfago e ao estudo radiográfico pneumoperitônio estava preseente (Figura 1). Foi indicada laparotomia exploradora

Trabalho realizado na Disciplina de Gastroenterologia Cirúrgica do Departamento de Cirurgia e Ortopedia do Hospital das Clínicas da Faculdade de Medicina de Botucatu - UNESP, Botucatu, SP, Brasil.

Correspondência: Dr. Rodrigo Severo de Camargo Pereira, e-mail: rodrigo_scp@uol.com.br que mostrou ruptura em pequena curvatura gástrica de aproximadamente $7 \mathrm{~cm}$ de extensão. A conduta adotada foi sutura da lesão em dois planos. A paciente recebeu alta hospitalar no $14^{\circ}$ pós-operatório após término da antibioticoterapia e recuperação da insuficiência respiratória causada pela pneumonia com bom seguimento ambulatorial a médio prazo.

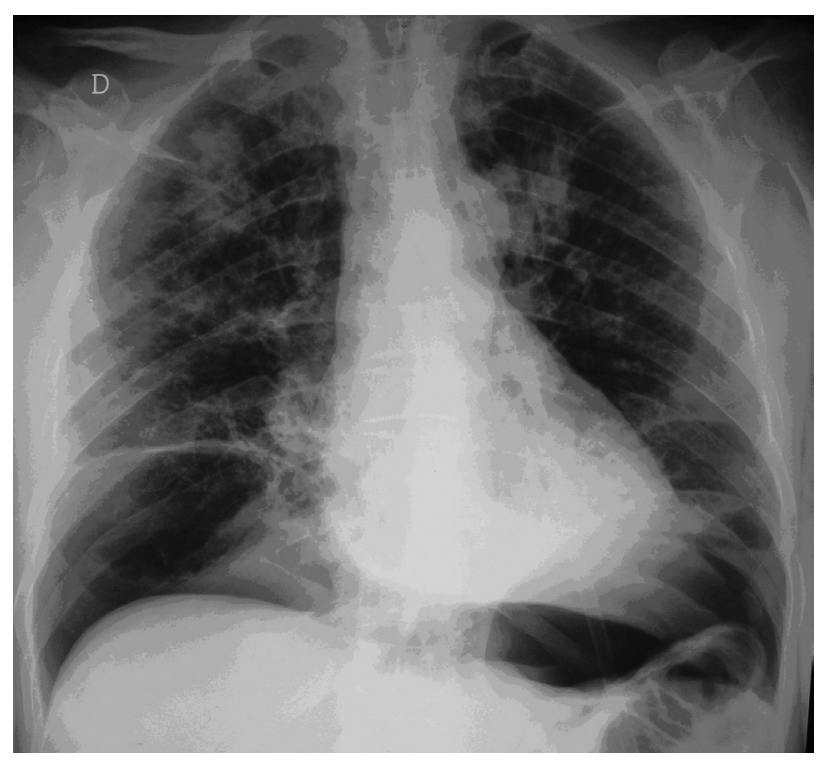

FIGURA 1 - Radiografia de tórax mostrando o pneumoperitônio 


\section{DISCUSSÃO}

As causas mais frequentes de ruptura gástrica por barotrauma encontrados na literatura estão relacionadas à reanimação cardiopulmonar, oxigenoterapia com cateter nasal e acidentes com mergulhadores ${ }^{2,3,7}$. Deve-se considerar a hipótese de ruptura gástrica em todo paciente submetido a recuperação cardio-respiratória que apresentar distensão abdominal refratária à sondagem nasogástrica ${ }^{5}$.

A lesão gástrica por barotrauma pode apresentar-se sob diversas formas, desde lacerações parciais da mucosa até ruptura completa da parede do estômago com pneumoperitônio hipertensivo, devido à distensão crescente do órgão associada a espasmo do piloro e angulação da cárdia, o que impede a saída do ar formando mecanismo de válvula ${ }^{7}$. Segundo Offerman et al, são necessários de 120 a $150 \mathrm{mmHg}$ de pressão para romper a parede gástrica normal ${ }^{5}$. No caso de pneumoperitônio hipertensivo, associado à distensão abdominal haverá hipotensão, sendo necessário punção abdominal para descompressão, seguido de laparotomia de emergência ${ }^{6}$.
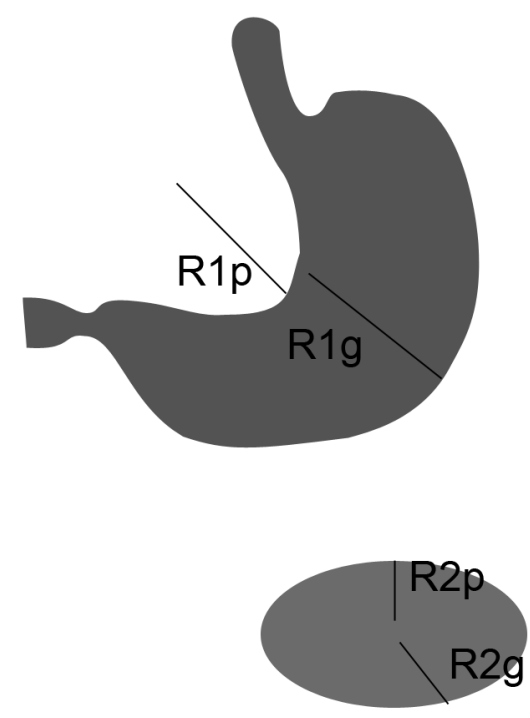

FIGURA 2 - Local preferencial para perfuração na pequena curvatura, onde o raio na visão coronal está direcionado para fora, enquanto que na grande curvatura, ambos direcionam-se para dentro, portanto, na pequena curvatura um dos raios tem sinal negativo
A lesão gástrica ocorre na pequena curvatura em aproximadamente $73 \%$ dos casos 7 e algumas séries de autópsias já revelaram a ocorrência de lacerações gástricas parciais em $10 \%$ dos pacientes submetidos à recuperação cárdio-respiratória, todas na pequena curvatura ${ }^{1}$.

A ocorrência preferencial da lesão gástrica por barotrauma na pequena curvatura já foi atribuída exclusivamente à Lei de Laplace, segundo a qual a pressão dentro de uma cavidade é diretamente proporcional à tensão exercida em sua parede e inversamente proporcional ao raio desta cavidade $(\mathrm{P}=\mathrm{T}(1 / \mathrm{R} 1+1 / \mathrm{R} 2)$.

Considerando que na pequena curvatura o raio na visão coronal está direcionado para fora e que na grande curvatura ambos direcionam-se para dentro, concluise que, na pequena curvatura, um dos raios tem sinal negativo. Portanto, neste caso, a soma dos raios será significativamente menor que na grande curvatura, exigindo assim maior tensão para resultar em um mesmo valor de P1(Figura 2).

No entanto, esta teoria não considera a heterogenicidade da parede gástrica. A pequena curvatura frequentemente apresenta largas pregas longitudinais em sua extensão com variações de espessura, sendo que as porções mais delgadas apresentam maior suscetibilidade aos aumentos de tensão ${ }^{4}$.

No presente caso foi optado por intervenção cirúrgica devido ao estado séptico da paciente e a incerteza diagnóstica, apesar de haver relatos na literatura ${ }^{7}$ de sucesso com o tratamento conservador das rupturas gástricas por barotrauma, baseado em antibioticoterapia de largo espectro e drenagem gástrica via sonda com aspiração contínua. Durante o procedimento verificou-se que a ruptura ocorreu na região mais frequentemente acometida (pequena curvatura) e o mecanismo de lesão foi devido a ventilação com pressão positiva no esôfago. Apesar da doença de base (tuberculose e pneumonia) a paciente apresentou boa evolução pós-operatória.

\section{CONCLUSÃo}

Apesar de rara, a ruptura gástrica por barotrauma deve ser sempre aventada quando, após reanimação cardiopulmonar, houver distensão abdominal refratária a sondagem nasogástrica. 
Pereira RSC, Arashiro RTG, Saad-Hossne R. Barogenic rupture of the stomach. ABCD Arq Bras Cir Dig 2008;21(3):144-6

ABSTRACT - Background - Barogenic rupture of the stomach is a rare cause of acute perforated abdomen generally treated by laparotomy and primary wound suture. The lesion of gastric mucosa may occur during cardiopulmonary resuscitation in 9 to $12 \%$ of cases. Case report - Woman was intubated in the fifth day of delivery due to nosocomial pneumonia. She underwent to abdominal distension associated to septic shock signs after the procedure. The abdominal X-ray showed pneumoperitoneum. She was submitted to laparotomy and a $7 \mathrm{~cm}$ rupture in the gastric small curvature was found. The lesion was treated by primary suture. The patient was discharged 14 days after the surgery, in the ending of pneumonia treatment. Conclusion - Besides rare, barogenic gastric rupture must be inquired when after cardiopulmonary resuscitation the patient presents abdominal distension ovenproof to nasogastric tube.

HEADINGS - Barotrauma. Stomach Rupture.

\section{REFERÊNCIAS}

1. Barker SJ, Karagianes T. Gastric barotrauma: a case report and theoretical considerations. Anesth Analg. 1985 Oct;64(10):1026-8.

2. Cole DS, Burcher SK. Accidental pneumatic rupture of oesophagus and stomach. Lancet. 1961 Jan 7;1(7167):24-5.

3. Darke SG, Bloomfield E. Case of complete gastric rupture complicating resuscitation. Br Med J. 1975 Aug 16;3(5980):414-5.

4. Kempen PM. Static and dynamic considerations in gastric barotrauma. Anesth Analg. 1986 May;65(5):540-1.
5. Offerman SR, Holmes JF, Wisner DH. Gastric rupture and massive pneumoperitoneum after bystander cardiopulmonary resuscitation. J Emerg Med. 2001 Aug;21(2):137-9.

6. Smally AJ, Ross MJ, Huot CP. Gastric rupture following bag-valve-mask ventilation. J Emerg Med. 2002 Jan;22(1):27-9.

7. Yeung P, Crowe P, Bennett M. Barogenic rupture of the stomach: a case for non-operative management. Aust N Z J Surg. 1998 Jan;68(1):76-7.

Fonte de financiamento: não há Conflito de interesse: não há Recebido para publicação: 15/12/2007 Aceito para publicação: 30/04/2008 